\title{
Total en bloc spondylectomy of the eleventh thoracic vertebra following denosumab therapy for the treatment of a giant cell tumor
}

\author{
GEN INOUE, TAKAYUKI IMURA, MASAYUKI MIYAGI, WATARU SAITO, \\ RYO TAZAWA, TOSHIYUKI NAKAZAWA and MASASHI TAKASO
}

Department of Orthopedic Surgery, Kitasato University School of Medicine, Sagamihara, Kanagawa 252-0374, Japan

Received December 31, 2016; Accepted June 21, 2017

DOI: $10.3892 / \mathrm{ol} .2017 .6655$

\begin{abstract}
Although denosumab has been reported to induce effective clinical results with respect to tumor shrinkage in a short-term follow-up clinical study, total spondylectomy is recognized as the treatment of choice for eradicating giant cell tumors (GCTs) of the spine. The present study reports the case involving a GCT in the 11th thoracic vertebra complicated by idiopathic scoliosis and treated using total en bloc spondylectomy (TES) with preoperative denosumab therapy. A 35-year-old woman received preoperative denosumab therapy for 8 months, followed by surgery using a computed tomography (CT)-based navigation system that optimized accuracy by recognizing the area of the detached parietal pleura, the irregular border of the collapsed vertebra, and the adjacent vertebra. Complete en bloc resection of the vertebra could be performed, suggesting denosumab can be an effective adjuvant therapy which can reduce the complexity of TES and CT-navigation system facilitated the safe use of this surgical method in a patient with idiopathic scoliosis.
\end{abstract}

\section{Introduction}

Giant cell tumors (GCTs) of the bone are aggressive benign primary bone neoplasms, which present mostly at the meta-epiphysis of long bones, causing extensive lytic lesions with an estimated incidence of 1.3 per million per year $(1,2)$. GCTs of the spine reportedly account for $2.7-6.5 \%$ of all GCTs in bone (3). Resection at an early stage remains the best strategy for treatment with a low recurrence rate $(4,5)$.

Correspondence to: Dr Gen Inoue, Department of Orthopedic Surgery, Kitasato University School of Medicine, 1-15-1 Kitasato, Minami, Sagamihara, Kanagawa 252-0374, Japan

E-mail: ginoue@kitasato-u.ac.jp

Key words: giant cell tumor, idiopathic scoliosis, eleventh thoracic vertebra, denosumab, total en bloc spondylectomy, navigation, histology
Although denosumab has been reported to induce effective clinical results with respect to tumor shrinkage in a short-term follow-up clinical study, total spondylectomy is recognized as the treatment of choice for eradicating GCTs of the spine (6). The present study reports a case involving a GCT in the eleventh thoracic vertebra, complicated by idiopathic scoliosis and treated using total en bloc spondylectomy (TES) following preoperative denosumab therapy for 8 months. Surgery was performed using a computed tomography (CT)-based navigation system that optimized accuracy by recognizing the area of the detached parietal pleura, the irregular border of the collapsed vertebra and the adjacent vertebra. Although recent studies have reported that preoperative denosumab treatment induces marked regression of GCTs of the spine, which subsequently permitted surgical resection that may otherwise have been unresectable had it not been for tumor shrinkage (7-10), there are no reports involving GCTs of the thoracic spine in a patient with idiopathic scoliosis treated by a posterior one-stage TES following preoperative denosumab therapy. In this case, analysis of the CT images after 8 months of preoperative denosumab therapy revealed the border between the vertebral body and soft tissue, indicating consolidation of the vertebral cortex. A CT-navigation system facilitated the safe use of the TES that followed.

\section{Case report}

A 35-year-old woman was referred to the Outpatient Department of Kitasato University Hospital (Kanagawa, Japan) for the evaluation of severe back pain, which started 4 months prior to her first visit to the hospital on 4th March 2015. The pain had gradually increased and persisted regardless of motion. The patient had been diagnosed with adolescent idiopathic scoliosis at 14 years old and the condition had never been treated. Although a neurological examination was negative, percussion tenderness around the thoracolumbar junction was noted.

Radiographic analysis revealed the collapse of the T11 vertebral body and idiopathic scoliosis with a Cobb angle measurement of $21^{\circ}$ (Fig. 1). The spinal CT revealed an osteolytic lesion involving the T11 vertebral body and the surrounding soft tissue, which had resulted in collapse of the 
vertebral body (Fig. 2). Magnetic resonance imaging (MRI) of the thoracolumbar spine showed the tumor extended toward the paravertebral soft tissue and into the left pedicle resulting in compression of the spinal cord (Fig. 3A). No additional sites of neoplasm were noted in the whole body. A needle biopsy was immediately performed to collect a sample that could be used to verify the diagnosis. Following fixation with $20 \%$ neural buffered formalin for $24 \mathrm{~h}$ at room temperature, paraffin-embedded, $4-\mu \mathrm{m}$-thick tissue sections from the biopsy specimen were stained with antibodies against vimentin (V9, Dako; Agilent Technologies, Inc., Santa Clara, CA, USA; cat. no. M6725), CD68 (PGM-1, Dako; Agilent Technologies, Inc.; cat. no. M0876), p53 (DO-7, Dako; Agilent Technologies, Inc.; cat. no. M7001) and MIB-1 (Dako; Agilent Technologies, Inc.; cat. no. M7240) antibodies for immunohistological analysis. For vimentin and MIB-1, slides were deparaffinized using PT-Link (Dako; Agilent Technologies, Inc.; cat. no. PT109) at $98^{\circ} \mathrm{C}$ for $20 \mathrm{~min}$, and then blocked with peroxidase-blocking reagent included in Envision FLEX Package High PH (Dako; Agilent Technologies, Inc.; cat. no. k8010) for 5 min. Dako AutoStainer plus (cat. no. S3400) was used with secondary antibody and visualization reagent included in Envision FLEX Package High PH (Dako; Agilent Technologies, Inc.; cat. no. k8010). For CD68 and p53, slides were deparaffinized with EZ Prep 10x (Ventana Medical Systems, Inc.; cat. no. 950-102), and then blocked with inhibitor reagent with $3 \% \mathrm{H}_{2} \mathrm{O}_{2}$ included in the i-View DAB Universal kit (Roche Diagnostics, Basel, Switzerland, 760-041). Autostainer Ventana BenchMark XT (Ventana Medical Systems, Inc., Tucson, AZ, USA) was used with the i-View DAB Universal kit. An Olympus BX51 polarizing microscope (Olympus Corporation, Tokyo, Japan) was used to observe the immunohistological staining results at a magnification of $x 40-400$. Pathological and immunohistochemical analyses confirmed a GCT characterized by multinucleate giant cells surrounded by neoplastic stromal cells (Fig. 4).

Based on evidence reported in a phase 2 clinical study, the patient was prescribed denosumab in weekly $120-\mathrm{mg}$ subcutaneous injections for 3 weeks, followed by monthly 120 -mg subcutaneous injections for 7 months (11). No adverse effects were observed. At 3 and 7 months after the start of denosumab therapy, thoracolumbar CT scans were performed and the imaging series showed the border of the vertebral body and soft tissue, including the spinal canal, indicating vertebral cortex consolidation (Fig. 5). The MRI showed that the intensity of vertebral body decreased to a level similar to normal vertebrae upon T2-weighted imaging, and compression of the spinal cord decreased owing to tumor shrinkage (Fig. 3B). The patient was scheduled for a TES of the T11 vertebra following 8 months of denosumab treatment. Preoperative angiography and embolization of the segmental artery from T10 to T12 was performed the day prior to surgery to reduce intraoperative bleeding (12). The TES for the resection of the T11 vertebra involved surgery via the posterior approach using transcranial electrical motor-evoked potentials for spinal cord neuromonitoring purposes. Immediately prior to surgery, a three-dimensional CT angiography image revealed that the anterior wall of the GCT had collapsed and the edge of the anterior wall was overlapping the adjacent vertebrae, which made the resection line irregular (Fig. 6). A CT-based
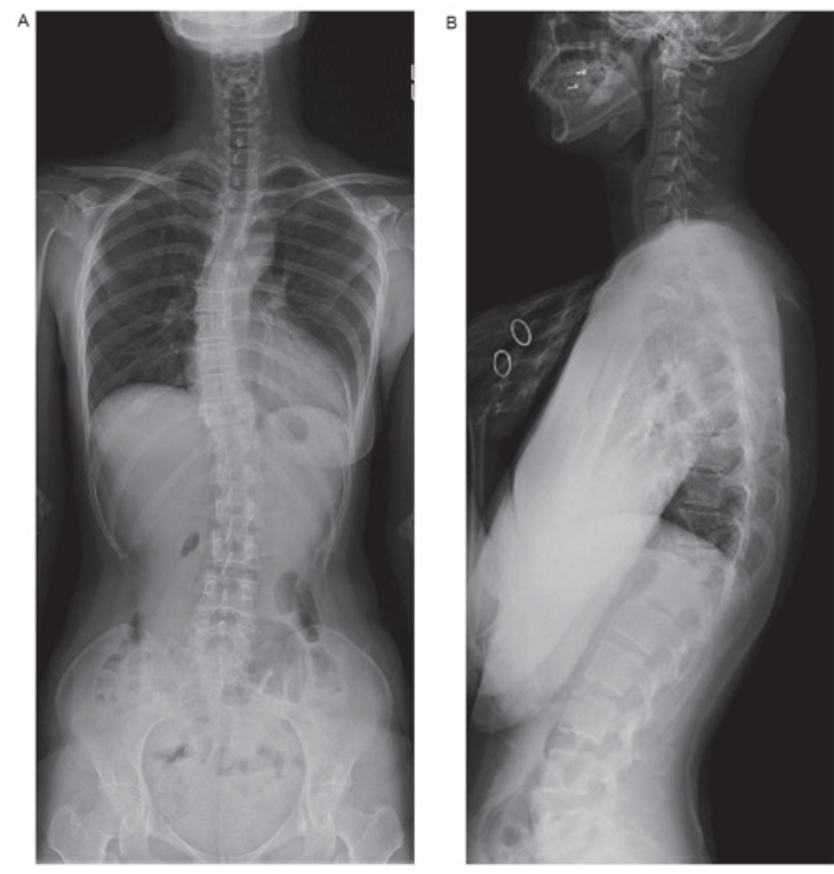

Figure 1. Plain radiographs of the whole spine prior to denosumab treatment. (A) Anteroposterior view. (B) Lateral view. The eleventh thoracic (T11) vertebral body collapsed and the idiopathic scoliosis present measured $21^{\circ}$ when using Cobb's angle.

navigation system (StealthStation; Medtronic Ltd., Memphis, TN, USA) was used for correct screw placement and to ensure the completion of an en bloc spondylectomy. Release of the border of the T11 vertebra was confirmed using the tip of the navigation probe. The TES was performed without any unexpected perioperative events (Fig. 7). Pathological analysis of the T11 vertebra demonstrated an absence of giant cells and stromal cells (Fig. 8). The patient is followed-up every 3 months and continues on monthly denosumab treatment without any complications or evidence of recurrence (Fig. 9).

Written informed patient consent was obtained for the publication of this study.

\section{Discussion}

A GCT of the spine is a rare entity, accounting for 2.7 to $6.5 \%$ of all types of GCTs in the bone (3), and its treatment remains a challenge. Currently, there is no consensus regarding the optimal treatment, which can be surgical or conservative (non-invasive) in nature, with or without adjuvant therapy (e.g., radiotherapy, arterial embolization, argon beam coagulation, cryotherapy, bisphosphonates or interferon). A treatment strategy should be decided on by taking into consideration multiple factors, including the age of the patient, lesion location, degree of tumor involvement, neurological status, feasibility of a wide resection and the presence of metastases or fractures (13). A complete surgical resection of the tumor is recognized as the ultimate goal when treating a GCT of the spine, as when it is performed efficiently, it can result in oncological control, a negated risk of local recurrence and the obviation of comorbidities associated with repeat surgery (13-15). 

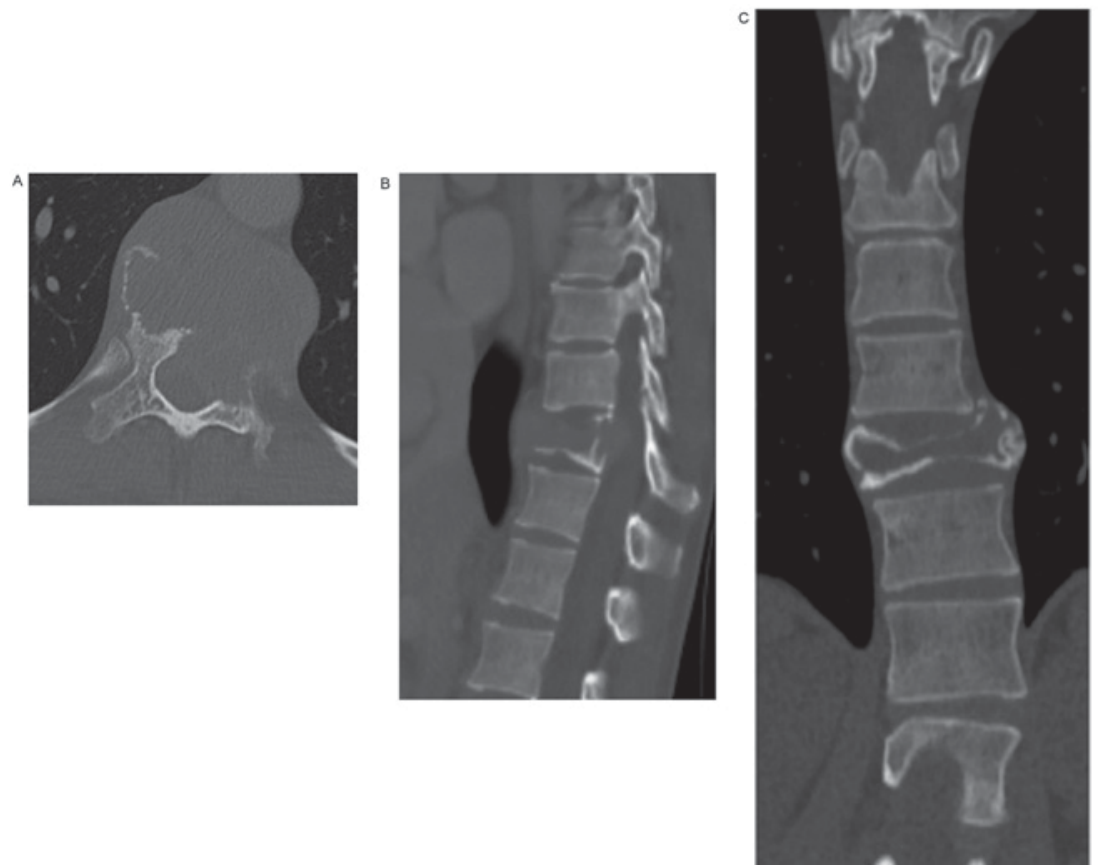

Figure 2. Computed tomography prior to denosumab treatment. (A) Axial view. (B) Sagittal view. (C) Coronal view. An osteolytic lesion involved the T11 vertebral body, which collapsed and expanded into the surrounding soft tissue.

A

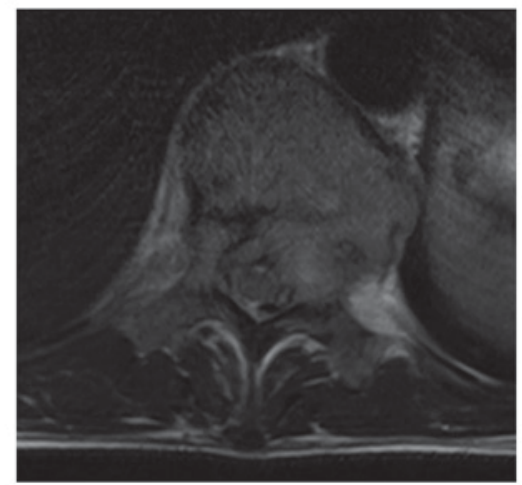

B

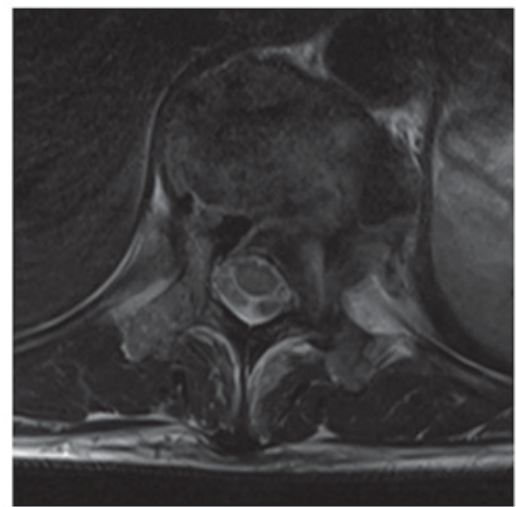

Figure 3. T2-weighted magnetic resonance images showing an axial view. (A) Prior to denosumab therapy. (B) At 8 months after denosumab therapy.

Intralesional curettage with adjuvant treatment is also an option, depending on the status of the patient, as it can provide good functional results. However, as high recurrence rates ranging from 36 to $49 \%$ have been reported (15), reoperation and radiotherapy treatment may be necessary if the treatment is not performed meticulously (13).

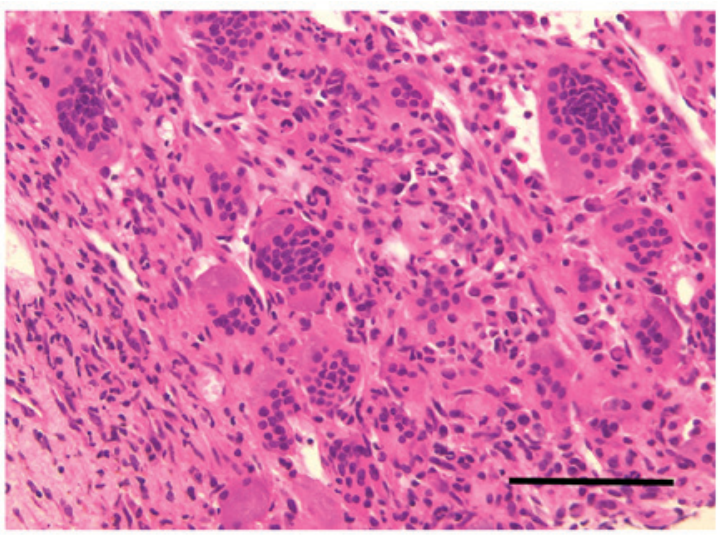

Figure 4. In hematoxylin and eosin staining, multinucleate giant cells were visible, surrounded by neoplastic stromal cells, and the diagnosis of a giant cell tumor was confirmed. Scale bar, $100 \mu \mathrm{m}$.

Recently, denosumab has been commonly used for the treatment of GCTs. In an open-label, phase 2 study, $86 \%$ of patients treated with denosumab therapy for 6 months were identified with an objective response, defined as $>90 \%$ elimination of giant cells on histological evaluation or no radiographic progression of the lesion (16). Another phase 2 study reported no disease progression in $96 \%$ of patients after a median follow-up time of 13 months (11). Based on these results, the U.S. Food and Drug Administration approved denosumab for the treatment of adults and skeletally mature adolescents with GCTs of the bone. In the present case, the vertebral cortex consolidation that was observed via CT imaging, following treatment for 8 months with denosumab, was marked. Recent studies have reported that preoperative denosumab treatment induced marked regression of GCTs of the spine, which subsequently permitted surgical resection on 
A

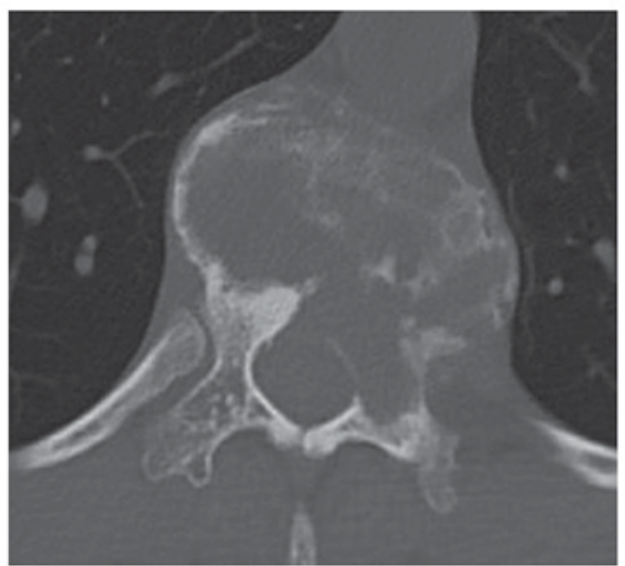

B

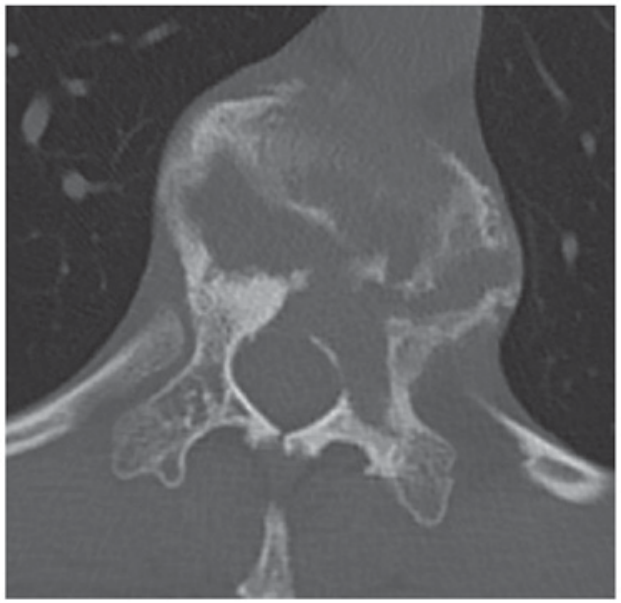

Figure 5. Computed tomography images captured during denosumab treatment. (A) At 3 months after starting denosumab therapy. (B) At 7 months after starting denosumab therapy. Consolidation of the vertebral cortex became increasingly visible as the border of the vertebral body and the soft tissue, including the spinal canal, were more easily distinguished compared with pretreatment images (Fig. 2A).
A

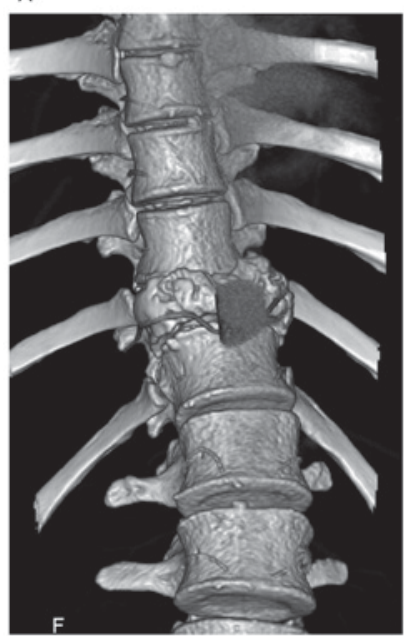

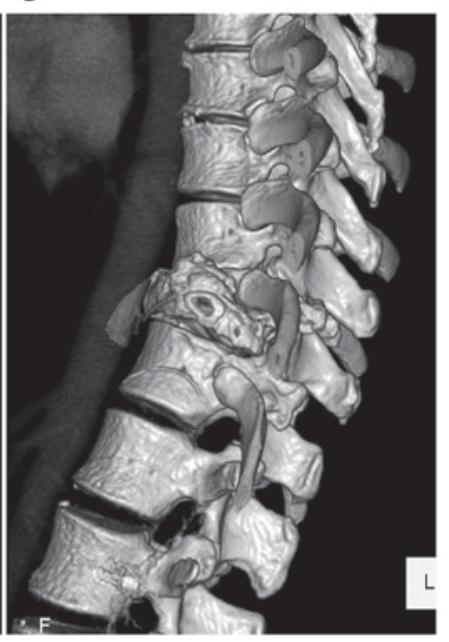

Figure 6. Three-dimensional computed tomography angiography images just prior to surgery. (A) An anterior view. (B) A lateral view. The anterior wall of T11 was collapsed and the edge of the anterior wall overlapped with adjacent vertebrae, which made the resection line irregular.

tumors that may otherwise have been unresectable had it not been for tumor shrinkage $(7,8,17)$. Agarwal et al $(17)$ reported

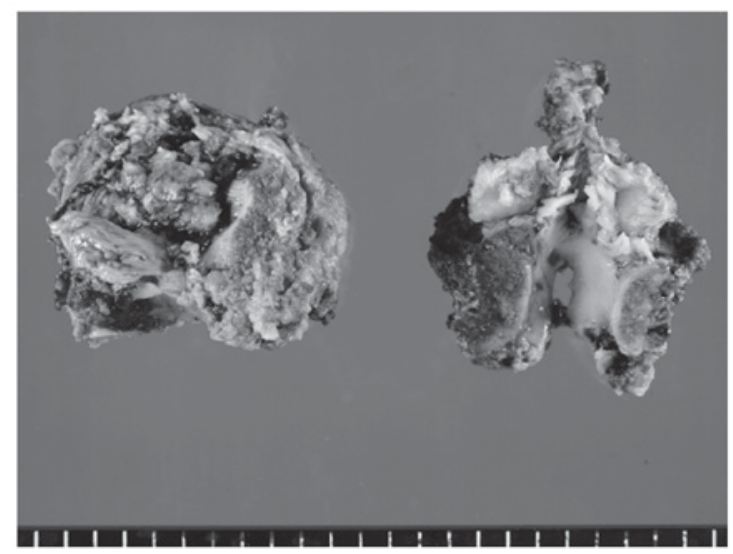

Figure 7. T11 vertebra was completely resected using a posterior approach and was separated into anterior and posterior parts.

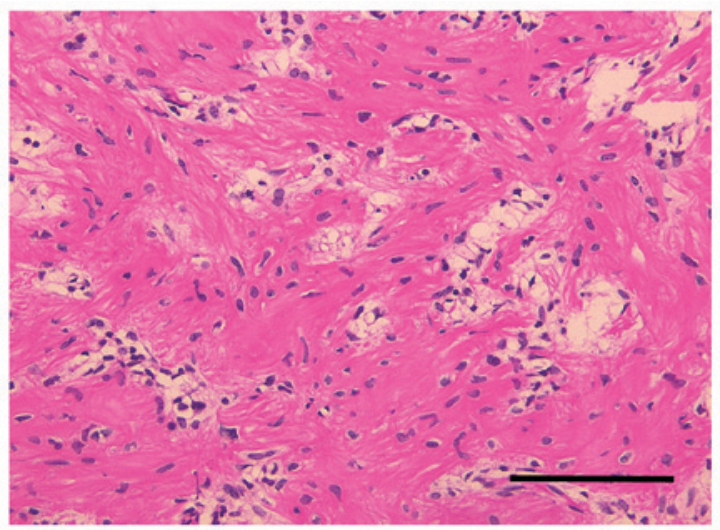

Figure 8. A pathological evaluation of the resected vertebra. Giant cells and stromal cells were not detected in the whole vertebra following 8 months of the denosumab therapy. Scale bar, $100 \mu \mathrm{m}$.

that a GCT at T6 had markedly shrunk after 13 months of denosumab therapy followed by a wide resection that included the lower lobe of the lung. Goldschlager et al (7) reported a multicenter, prospective series of 5 cases of GCT of the spine treated preoperatively with denosumab; following a mean treatment period of 6 months, denosumab reduced the tumor size by $10-40 \%$ compared with the size before treatment (7). de Carvalho Cavalcante et al (8) reported a case of TES for L4, following preoperative denosumab treatment for 6 months, which showed tumor regression of $\sim 90 \%$ with vertebral body calcification. Therefore, total resection subsequent to preoperative denosumab therapy may be one therapeutic option available for the management of aggressive and locally advanced GCTs of the spine.

The duration of denosumab treatment is not only controversial prior to surgery, but also postoperatively. Following surgery, the present patient continued to receive denosumab, even after a histological evaluation of the removed vertebra, which did not contain any giant cells or stromal cells, similar to the case reported by de Carvalho Cavalcante et al (8). The aforementioned case involved the continuation of denosumab once every 3 months after surgery without any evidence of tumor recurrence (17), whereas other studies were of patients who stopped denosumab therapy prior to surgery (7). 

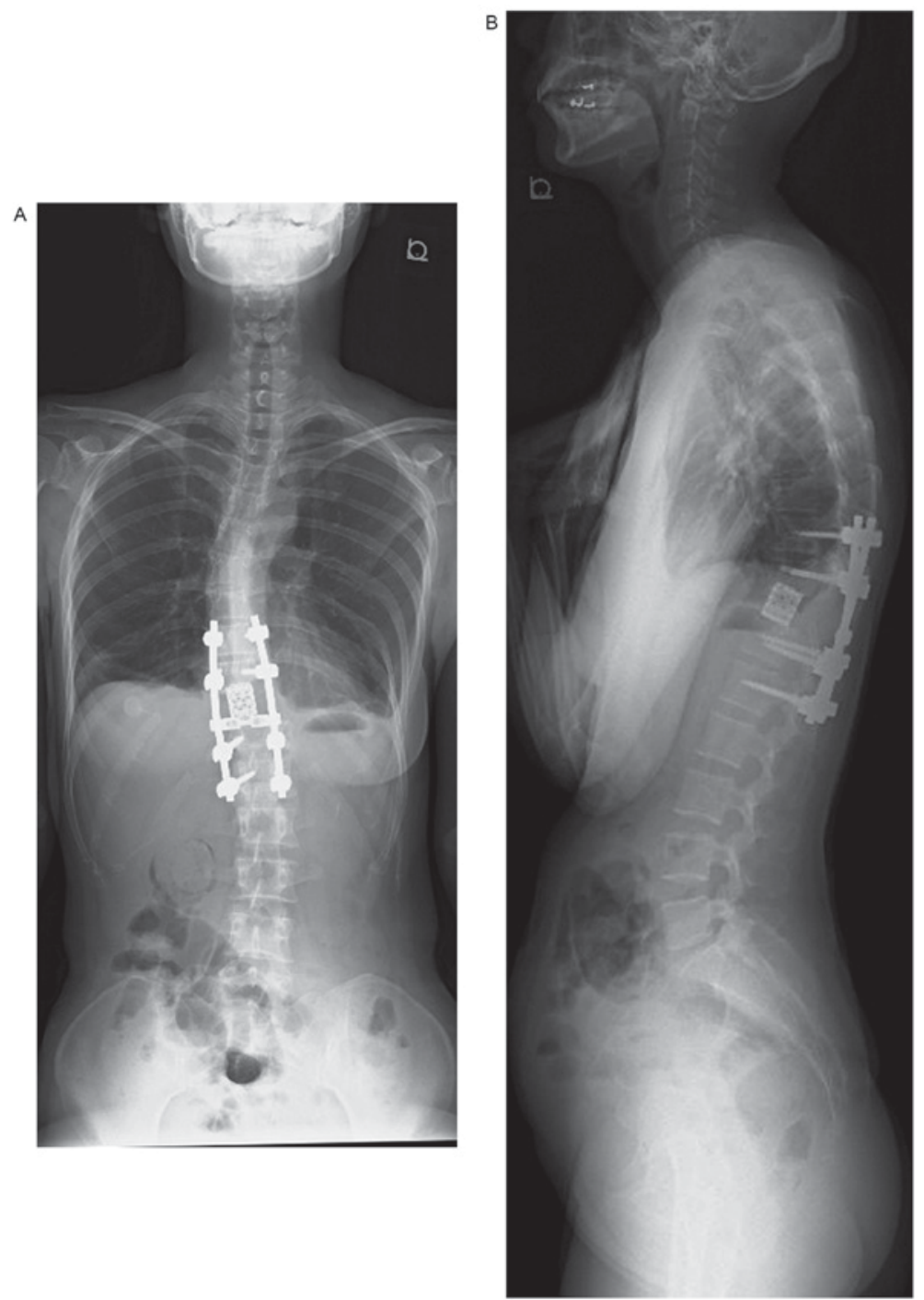

Figure 9. Plain radiographs of the whole spine 3 months after a total en bloc spondylectomy. (A) An anteroposterior view. (B) A lateral view.

$\mathrm{Xu}$ et al (6) reviewed 102 patients who underwent TES and reported that long-term postoperative bisphosphonate treatment significantly reduced tumor recurrence rate, as assessed by multivariate analysis (6). After TES, and in the absence of postoperative bisphosphonate treatment, $>50 \%$ of patients experienced tumoral relapse within a mean follow-up period of 39.9 months, suggesting that spinal GCTs can indeed recur after TES. By contrast, Müller et al (9) reported 18 cases of GCTs in the extremities or sacrum of patients who were treated postoperatively with monthly denosumab for 6 months and who remained free of recurrent disease after a mean follow-up period of 22 months (9). Based on this evidence, and with the informed consent of all patients, the authors now use denosumab prophylactically after TES. Furthermore, the treatment plan involves discontinuing denosumab 6 months after surgery, and follow-up spinal radiographs and CT are performed every 3 months and 1 year after surgery, respectively, to evaluate for potential disease recurrence. However, a longer follow-up period is necessary.

Recently, 3 cases of high-grade sarcoma arising in GCTs of the bone in patients treated with denosumab have been reported $(18,19)$. The potential association between sarcomatous transformations of GCT and osteosarcoma in patients receiving denosumab therapy is unclear, owing at least in part to the limited published data for this population. Long-term follow-up is therefore necessary. The clinical outcome, following completion of an adequate duration of denosumab treatment, remains uncertain. Further evidence to support how to use denosumab after the resection of spinal GCT is definitely required.

In terms of the present case, a CT-based navigation system was used as the patient presented with idiopathic scoliosis, the affected vertebra was rotated and collapsed, and the edge of the tumor had overlapped the adjacent vertebrae. Recently, clinical studies have demonstrated that CT-based navigation systems are useful as an assistance device to optimize the accuracy of pedicle screw placement during surgery in patients with scoliosis (20-22). Muscloskeletal oncologists also use CT-based navigation systems for pelvic and sacral tumor resection, suggesting its potential to increase the accuracy of tumor resections of anatomical and/or surgical complexity $(23,24)$. For malignant bone tumors of the metaphyses of the long bones, or in iliac bones, CT-based navigation is reported to 
facilitate precise planning and execution of joint-preserving tumor resections and reconstructions, resulting in good functional and oncological outcomes (25-27). Tian et al (28) used CT-based navigation in posterior decompression surgery for thoracic ossification of the posterior longitudinal ligament (OPLL) in order to identify the border of the vertebrae and part of the OPLL (28). In the present case, by positioning the tip of the navigation probe at the surface of the vertebra or osteotomy line, the surgeons were able to recognize the area of detached parietal pleura, the irregular border of the collapsed T11 vertebra and the adjacent vertebrae, making it possible to insert the chisel at an angle and in the necessary direction for optimizing accuracy. As a result, the TES was performed in a safe manner, particularly considering the collapsed and expanded GCT, and the accuracy of screw insertion was optimized.

In conclusion, the present study reports a case of a GCT in the spine of a patient with idiopathic scoliosis who was treated using a TES with the aid of CT-based navigation following 8 months of denosumab treatment. Denosumab can be an effective adjuvant therapy and it can reduce the complexity of TES, a major surgical procedure used for the effective treatment of GCTs of the spine.

\section{Acknowledgements}

The authors would like to thank Mr. Tatsuru Kuba, (Department of Pathology, Kitasato University Hospital) and Professor Hiroyuki Takahashi, (Kitasato University School of Allied Health Sciences) for their technical support in pathological evaluations.

\section{References}

1. Rockberg J, Bach BA, Amelio J, Hernandez RK, Sobocki P, Engellau J, Bauer HC and Liede A: Incidence Trends in the diagnosis of giant cell tumor of bone in Sweden since 1958. J Bone Joint Surg Am 97: 1756-1766, 2015.

2. Gong L, Liu W, Sun X, Sajdik C, Tian X, Niu X and Huang X: Histological and clinical characteristics of malignant giant cell tumor of bone. Virchows Arch 460: 327-334, 2012.

3. Shimada Y, Hongo M, Miyakoshi N, Kasukawa Y, Ando S, Itoi E and Abe E: Giant cell tumor of fifth lumbar vertebrae: Two case reports and review of the literature. Spine J 7: 499-505, 2007.

4. Niu X, Zhang Q, Hao L, Ding Y, Li Y, Xu H and Liu W: Giant cell tumor of the extremity: Retrospective analysis of 621 Chinese patients from one institution. J Bone Joint Surg Am 94: 461-467, 2012.

5. Wang K, Zhu B, Yang S, Liu Z, Yu M and Liu X: Primary diffuse-type tenosynovial giant cell tumor of the spine: A report of 3 cases and systemic review of the literature. Turk Neurosurg 24: 804-813, 2014.

6. Xu W, Li X, Huang W, Wang Y, Han S, Chen S, Xu L, Yang X, Liu $\mathrm{T}$ and Xiao J: Factors affecting prognosis of patients with giant cell tumors of the mobile spine: Retrospective analysis of 102 patients in a single center. Ann Surg Oncol 20: 804-810, 2013.

7. Goldschlager T, Dea N, Boyd M, Reynolds J, Patel S, Rhines LD, Mendel E, Pacheco M, Ramos E, Mattei TA and Fisher CG: Giant cell tumors of the spine: Has denosumab changed the treatment paradigm? J Neurosurg Spine 22: 526-533, 2015.

8. de Carvalho Cavalcante RA, Silva Marques RA, dos Santos VG, Sabino E, Fraga AC Jr, Zaccariotti VA, Arruda JB and Fernandes YB: Lumbar spondylectomy for giant cell tumor after denosumab therapy. Spine (Phila Pa 1976) 41: E178-E182, 2016.

9. Müller DA, Beltrami G, Scoccianti G, Campanacci DA, Franchi A and Capanna R: Risks and benefits of combining denosumab and surgery in giant cell tumor of bone-a case series. World J Surg Oncol 14: 281, 2016
10. Deveci MA, Paydaş S, Gönlüşen G, Özkan C, Biçer ÖS and Tekin M: Clinical and pathological results of denosumab treatment for giant cell tumors of bone: Prospective study of 14 cases. Acta Orthop Traumatol Turc 51: 1-6, 2017.

11. Chawla S, Henshaw R, Seeger L, Choy E, Blay JY, Ferrari S, Kroep J, Grimer R, Reichardt P, Rutkowski P, et al: Safety and efficacy of denosumab for adults and skeletally mature adolescents with giant cell tumour of bone: Interim analysis of an open-label, parallel-group, phase 2 study. Lancet Oncol 14: 901-908, 2013.

12. Tomita K, Kawahara N, Murakami H and Demura S: Total en bloc spondylectomy for spinal tumors: Improvement of the technique and its associated basic background. J Orthop Sci 11: $3-12,2006$.

13. Fisher CG, Saravanja DD, Dvorak MF, Rampersaud YR, Clarkson PW, Hurlbert J, Fox R, Zhang H, Lewis S, Riaz S, et al: Surgical management of primary bone tumors of the spine: Validation of an approach to enhance cure and reduce local recurrence. Spine (Phila Pa 1976) 36: 830-836, 2011.

14. Bandiera S, Boriani S, Donthineni R, Amendola L, Cappuccio M and Gasbarrini A: Complications of en bloc resections in the spine. Orthop Clin North Am 40: 125-131, vii, 2009.

15. Arbeitsgemeinschaft Knochentumoren; Becker WT, Dohle J, Bernd L, Braun A, Cserhati M, Enderle A, Hovy L, Matejovsky Z, Szendroi M, et al: Local recurrence of giant cell tumor of bone after intralesional treatment with and without adjuvant therapy. J Bone Joint Surg Am 90: 1060-1067, 2008.

16. Thomas D, Henshaw R, Skubitz K, Chawla S, Staddon A, Blay JY, Roudier M, Smith J, Ye Z, Sohn W, et al: Denosumab in patients with giant-cell tumour of bone: An open-label, phase 2 study. Lancet Oncol 11: 275-280, 2010.

17. Agarwal A, Larsen BT, Buadu LD, Dunn J, Crawford R, Daniel J and Bishop MC: Denosumab chemotherapy for recurrent giant-cell tumor of bone: A case report of neoadjuvant use enabling complete surgical resection. Case Rep Oncol Med 2013: 496351, 2013.

18. Aponte-Tinao LA, Piuzzi NS, Roitman P and Farfalli GL: A high-grade sarcoma arising in a patient with recurrent benign giant cell tumor of the proximal tibia while receiving treatment with denosumab. Clin Orthop Relat Res 473: 3050-3055, 2015.

19. Broehm CJ, Garbrecht EL, Wood J and Bocklage T: Two cases of sarcoma arising in giant cell tumor of bone treated with denosumab. Case Rep Med 2015: 767198, 2015.

20. Kotani Y, Abumi K, Ito M, Takahata M, Sudo H, Ohshima S and Minami A: Accuracy analysis of pedicle screw placement in posterior scoliosis surgery: Comparison between conventional fluoroscopic and computer-assisted technique. Spine (Phila Pa 1976) 32: 1543-1550, 2007

21. Sakai Y, Matsuyama Y, Nakamura H, Katayama Y, Imagama S, Ito $\mathrm{Z}$ and Ishiguro N: Segmental pedicle screwing for idiopathic scoliosis using computer-assisted surgery. J Spinal Disord Tech 21: 181-186, 2008.

22. Takahashi J, Hirabayashi H, Hashidate H, Ogihara N and Kato H: Accuracy of multilevel registration in image-guided pedicle screw insertion for adolescent idiopathic scoliosis. Spine (Phila PA 1976) 35: 347-352, 2010.

23. Krettek C, Geerling J, Bastian L, Citak M, Rücker F, Kendoff D and Hüfner T: Computer aided tumor resection in the pelvis. Injury 35: S-A79-S-A83, 2004.

24. HüfnerT, Kfuri M Jr, Galanski M,Bastian L,Loss M, Pohlemann T and Krettek C: New indications for computer-assisted surgery: Tumor resection in the pelvis. Clin Orthop Relat Res 426: 219-225, 2004.

25. Wong KC and Kumta SM: Joint-preserving tumor resection and reconstruction using image-guided computer navigation. Clin Orthop Relat Res 471: 762-773, 2013.

26. Cho HS, Oh JH, Han I and Kim HS: Joint-preserving limb salvage surgery under navigation guidance. J Surg Oncol 100: 227-232, 2009.

27. Li J, Wang Z, Guo Z, Chen GJ, Yang M and Pei GX: Irregular osteotomy in limb salvage for juxta-articular osteosarcoma under computer-assisted navigation. J Surg Oncol 106: 411-416, 2012.

28. Tian W, Weng C, Liu B, Li Q, Sun YQ, Yuan Q, Zhang B, Wang YQ and He D: Intraoperative 3-dimensional navigation and ultrasonography during posterior decompression with instrumented fusion for ossification of the posterior longitudinal ligament in the thoracic spine. J Spinal Disord Tech 26: E227-E234, 2013. 